\title{
COMPARATIVE OUTCOMES OF BLADDER CANCER IN PATIENTS UNDER 40 YEARS OF AGE
}

Rebecca Tregunna ${ }^{1}$, Mark Feneley ${ }^{2}$, Alex Freeman ${ }^{3}$, and Dan Wood ${ }^{2}$

${ }^{1}$ Department of Urology, Heartlands Hospital, University Hospitals Birmingham NHS Foundation Trust, Bordesley Green East, Birmingham,

${ }^{2}$ Department of Urology, University College Hospital, London, UK.

${ }^{3}$ Department of Histopathology, University College Hospital, London, UK.

Corresponding Author: rebecca.tregunna@doctors.org.uk

Submitted: June 18, 2019. Accepted: July 31, 2019. Published: October 28, 2019.

\section{ABSTRACT}

\section{Background and Objectives}

Bladder tumours are rare in young patients. Consequently, the literature is sparse and studies provide conflicting reports on clinicopathological data and patient outcomes. This study examines, to our knowledge, the largest UK series of patients aged less than 40 years diagnosed with bladder cancer, and examines their risks and outcomes.

\section{Material and Methods}

A prospectively recorded database (2008-2018) was used to identify patients aged under 40 years diagnosed with bladder cancer. Data were retrospectively analyzed. Patients were then sub-divided into 2 groups based on age: group 1 was aged below 20 years and group 2 was aged 20-39 years.

\section{Results}

A total of 27 patients were identified with a median age of 34 years (range 14-39). Male to female ratio was 2.86:1. Median follow-up was 41.5 months (range 3-108). 61.9\% presented with visible hematuria and $54.2 \%$ had one or more risk factors such as cigarette smoking. A total of $96.3 \%$ of patients had urothelial carcinoma and $92.3 \%$ of these were non-muscle invasive tumours. $7.7 \%$ presented with muscle invasive disease with $3.8 \%$ having positive nodes at diagnosis. Patients with non-muscle invasive urothelial tumours were risk-stratified according to the EAU-Guidelines Panel risk grouping with $39.1 \%$ low-risk, $4.3 \%$ intermediate-risk and $56.5 \%$ high-risk of recurrence and/or progression. During follow-up 30.4\% recurred and $4.3 \%$ progressed to invasive disease. $23.1 \%$ underwent cystectomy and overall $11.5 \%$ died during follow-up, all due to metastatic disease. Patients in group one showed a statistically significant incidence of lower-grade disease at diagnosis with lower risk-group stratification but there was no significant difference in other parameters.

\section{Conclusion}

The majority of young patients in our series presented with non-muscle invasive urothelial bladder tumours but a significant proportion of these had high-risk disease. Some patients presented with aggressive, muscle invasive bladder cancer and consequently bladder cancer remains an important differential diagnosis in symptomatic patients regardless of age. 
The incidence of bladder cancer increases with age; with a peak incidence in the sixth decade of life. ${ }^{1}$ Only $1.0-2.4 \%$ of bladder tumours are found in patients aged below 40 years, ${ }^{2}$ with $0.4 \%$ diagnosed in patients under the age of 20 years. ${ }^{3}$ Anecdotal evidence and early case reports suggest young patients with bladder cancer have low-grade disease and the majority tend not to progress to more advanced stages. ${ }^{3}$ However, several retrospective studies have demonstrated outcomes more in keeping with older patients. The definition of 'young' has also been questioned with variation between studies leading to potential difficulty with data interpretion. ${ }^{4}$ This study evaluated the clinicopathological data and outcomes for patients aged 40 years or less diagnosed with a bladder tumour from a single institution over 10 years.

\section{METHODS}

A 10-year prospectively recorded database from 2008-2018 from a single large UK institution was consulted to identify patients $<40$ years of age who had been diagnosed with bladder cancer. Data were retrospectively audited. Clinic letters, operation notes, histopathology and radiology reports as well as multidisciplinary meeting outcomes were reviewed to provide data on demographics, risk factors, presentation characteristics, risk stratification, and follow-up. Histological grading of tumours was taken from histopathology reports at the time of diagnosis according to both 1973 and 2004 WHO classifications, and staging was according to the TNM classification.

Patients were subdivided into two groups based on age. Group one were aged less than 20 years and group two were aged between 20 and 39 years. This arbitrary age division was used to allow comparison with previous studies in the literature that had found a difference between these age groups in terms of outcomes and molecular pathology. ${ }^{5-7}$

Patients with non-muscle invasive urothelial tumours, were stratified acrording to the European Association of Urology (EAU) Guidelines-Panel risk grouping which takes into account the probabilities of both recurrence and progression by using prognostic data of number of tumours, tumour diameter, prior recurrence rate, grade, stage and presence of carcinoma in situ (CIS) (Table 1). ${ }^{8}$

Statistical analysis was performed using an online statistical programme (http:// vassarstats.net/ index.html). Fisher exact probability test with the Freeman-Halton extension was used to analyze categorical variables and the Kaplan-Meier survival analysis was used to calculate survival. $\quad \mathrm{P}<0.05$ was considered clinically significant.

\section{Demographics}

A total of 27 patients were identified with a median age of 34 years (range 14-39). Male to female ratio was 2.86:1. Median follow-up was 41.5 months (range 3-108). One patient was lost to follow-up having moved overseas three months following diagnosis.

\section{Presentation and Risk Factors}

Data were available for 21 patients and are presented in Table 2. A total of $13(61.9 \%)$ presented with visible hematuria, $3(14.3 \%)$ presented with recurrent urinary tract infections (UTI), $2(9.5 \%)$ presented with lower urinary tract symptoms (LUTS), 2 (9.5\%)

TABLE 1 EAU Guidelines Panel Risk Group Stratification ${ }^{8}$

(Low-grade is combination of G1 and some G2 and high-grade is combination of some G2 and all G3)

\begin{tabular}{|c|c|}
\hline Tumour Risk Group Stratification & Tumour Characteristics \\
\hline Low-risk & $\begin{array}{l}\text { Primary, solitary, }<3 \mathrm{~cm} \text { PUNLMP or low-grade } \mathrm{pTa} \text {, } \\
\text { with no CIS }\end{array}$ \\
\hline Intermediate-risk & All tumours not in low or high-risk category \\
\hline \multirow[t]{3}{*}{ High-risk } & $\begin{array}{l}\text { Any of: } \\
\text { High-grade } \\
\text { CIS } \\
\text { T1 } \\
\text { All of: Multiple, recurrent and }>3 \mathrm{~cm} \text { low-grade Ta }\end{array}$ \\
\hline & Subset of Highest-risk tumours \\
\hline & $\begin{array}{l}\text { High-grade T1 + CIS } \\
\text { Multiple and/ or large and/ or recurrent high-grade } \\
\text { T1 } \\
\text { Lymphovascular invasion }\end{array}$ \\
\hline
\end{tabular}


were incidental findings on an ultrasound scan and one $(4.8 \%)$ presented with deep vein thrombosis and was found to have node-positive disease at diagnosis.

One or more risk factors for bladder cancer were found in 13 out of $24(54.2 \%)$ patients for whom data were available. A total of 13 (54.2\%) were smokers, $1(4.2 \%)$ additionally had a history of cyclophosphamide exposure and 1 patient (4.2\%) also had had a previous nephroureterectomy for urothelial carcinoma of the renal pelvis (see Table 2).

\section{Stage, Grade and EAU Guidelines-Panel Risk Group Stratification}

Following initial TURBT 26 (96.3\%) had histology consistent with urothelial carcinoma of the bladder (UCB); data for these patients are presented in Table 3. One patient $(3.7 \%)$ had histology that demonstrated epithelioid angiosarcoma.

Of the patients with $\mathrm{UCB}(\mathrm{n}=26)$ one patient $(3.8 \%)$ presented with muscle invasive node-negative UCB and another $(3.8 \%)$ presented with muscle invasive node-positive disease. The remainder of the patients $(n=24)$ presented with non-muscle invasive UCB. Two patients $(7.7 \%)$ presented with variant histology (squamous differentiation in one patient with non-muscle invasive disease and micropapillary variant in the patient with muscle invasive, node-negative disease). One patient $(3.8 \%)$ with non-muscle invasive UCB presented with a synchronous renal pelvis tumour and underwent nephroureterectomy with the histology demonstrating G3 pT3 urothelial carcinoma. The patient with muscle invasive, node-negative UCB also had a G3 pT2 urothelial carcinoma of the ureter.

The 24 non-muscle invasive urothelial tumours, were stratified according to the EAU Guidelines-Panel risk grouping.

TABLE 2 Demographics, Presentation, and Risk Factors for Patients with Bladder Tumours

(Data for presentation not available for one patient in the younger group and five patients in the older group. Data for risk factors not available for one patient in the younger group and two patients in the older group).

\begin{tabular}{|c|c|c|c|c|}
\hline Characteristic & $\begin{array}{c}\text { Group One } \\
\text { Age }<20 \text { at } \\
\text { diagnosis } \\
n=4\end{array}$ & $\begin{array}{c}\text { Group Two } \\
\text { Age 20-39 at } \\
\text { diagnosis } \\
n=23\end{array}$ & $\begin{array}{l}\text { Total } \\
\mathbf{n}=27\end{array}$ & p value \\
\hline \multicolumn{5}{|l|}{ Demographic } \\
\hline $\begin{array}{l}\text { Median age at } \\
\text { diagnosis (range) }\end{array}$ & $16.5(14-19)$ & $35(23-39)$ & $34(14-39)$ & \\
\hline Male & 2 & 18 & 20 & \multirow[t]{2}{*}{0.545} \\
\hline Female & 2 & 5 & 7 & \\
\hline \multicolumn{5}{|l|}{ Presentation (\%) } \\
\hline Visible hematuria & $1(33.3)$ & $12(66.7)$ & $13(61.9)$ & \\
\hline Recurrent UTI & $2(66.7)$ & $1(5.6)$ & $3(14.3)$ & \\
\hline LUTS & 0 & $2(11.1)$ & $2(9.5)$ & \\
\hline Incidental finding & 0 & $2(11.1)$ & $2(9.5)$ & \\
\hline DVT & 0 & $1(5.6)$ & $1(4.8)$ & \\
\hline \multicolumn{5}{|l|}{ Risk Factors (\%) } \\
\hline Smoking & 0 & $13(61.9)$ & $13(54.2)$ & \multirow{4}{*}{0.082} \\
\hline Cyclophosphamide & 0 & $1(4.8)$ & $1(4.2)$ & \\
\hline $\begin{array}{l}\text { Urothelial carcinoma } \\
\text { of the renal pelvis }\end{array}$ & 0 & $1(4.8)$ & $1(4.2)$ & \\
\hline None & $3(100.0)$ & $8(38.1)$ & $11(45.8)$ & \\
\hline
\end{tabular}


TABLE 3 WHO 1973/2004 Grading and TNM Staging of Patients with Urothelial Carcinoma of the Bladder Following Initial TURBT.

(Data on grade is not available for one patient who had non-muscle invasive UCB from the older group, p value for grade based on 2004 WHO grading).

\begin{tabular}{|c|c|c|c|c|}
\hline Histology & $\begin{array}{c}\text { Group One } \\
\text { Age }<20 \text { at } \\
\text { diagnosis } \\
n=4 \\
\end{array}$ & $\begin{array}{c}\text { Group Two } \\
\text { Age 20-39 at } \\
\text { diagnosis } \\
n=22\end{array}$ & $\begin{array}{l}\text { Total } \\
n=26\end{array}$ & p value \\
\hline \multicolumn{5}{|l|}{ Grade (\%) } \\
\hline PUNLMP & 0 & $1(4.8)$ & $1(4.0)$ & \multirow{6}{*}{0.026} \\
\hline G1 & $1(25.0)$ & $1(4.8)$ & $2(8.0)$ & \\
\hline $\begin{array}{l}\text { G2 (Low } \\
\text { grade) }\end{array}$ & $3(75.0)$ & $5(23.8)$ & $8(32.0)$ & \\
\hline $\begin{array}{l}\text { G2 (High } \\
\text { grade) }\end{array}$ & 0 & $4(19.0)$ & $4(16.0)$ & \\
\hline G3 & 0 & $8(38.1)$ & $8(32.0)$ & \\
\hline CIS & 0 & $2(9.5)$ & $2(8.0)$ & \\
\hline \multicolumn{5}{|l|}{ Stage (\%) } \\
\hline $\mathrm{Ta}$ & $4(100.0)$ & $12(54.5)$ & $16(61.5)$ & \multirow{4}{*}{0.775} \\
\hline $\mathrm{T} 1$ & 0 & $6(27.3)$ & $6(23.1)$ & \\
\hline Tis only & 0 & $2(9.1)$ & $2(7.7)$ & \\
\hline $\begin{array}{l}\text { Muscle } \\
\text { invasive (T2a } \\
\text { at least) }\end{array}$ & 0 & $2(9.1)$ & $2(7.7)$ & \\
\hline N0 & $4(100.0)$ & $21(95.5)$ & $25(96.2)$ & \multirow[t]{2}{*}{1.000} \\
\hline $\mathrm{N}+$ & 0 & $1(4.5)$ & $1(3.8)$ & \\
\hline
\end{tabular}


One patient had insufficient data to risk stratify, therefore of the 23 other cases, 9 (39.1\%) were stratified as low-risk, 1 (4.3\%) was intermediate-risk and $13(56.5 \%)$ were high-risk with 5 of these $(21.7 \%)$ being subdivided into the highest-risk group (see Table 4).

\section{Recurrence and Progression}

These data are presented in Table 4. Of the nonmuscle invasive bladder tumours follow-up was not possible in one patient after 3 months. Seven of these remaining patients $(30.4 \%)$ had recurrences during the follow-up period. A total of 5 of these $(71.4 \%)$ were originally stratified as high-risk, the other 2 (28.6\%) were originally stratified as intermediate or low-risk. The median time to recurrence was 8 months (range 5-37).

TABLE 4 EAU Guidelines-Panel Risk Group Stratification, Recurrence, Progression, Cystectomy and Death Rates for Patients with Non-muscle Invasive UCB.

(Data not available for risk group stratification for one patient from the older group. Data on recurrence, progression, cystectomy and death not available for one patient from the older group who was lost to follow-up).

\begin{tabular}{|c|c|c|c|c|}
\hline & $\begin{array}{c}\text { Group One } \\
\text { Age }<20 \text { at } \\
\text { diagnosis } \\
n=4\end{array}$ & $\begin{array}{c}\text { Group Two } \\
\text { Age 20-39 at } \\
\text { diagnosis } \\
n=20\end{array}$ & $\begin{array}{l}\text { Total } \\
\mathbf{n}=24\end{array}$ & p value \\
\hline \multicolumn{5}{|l|}{$\begin{array}{l}\text { EAU Risk Group } \\
(\%)\end{array}$} \\
\hline Low & $3(75.0)$ & $6(31.6)$ & $9(39.1)$ & \multirow{3}{*}{0.009} \\
\hline Intermediate & $1(25.0)$ & 0 & $1(4.3)$ & \\
\hline High & 0 & $13(68.4)$ & $13(56.5)$ & \\
\hline Recurrence (\%) & $1(25.0)$ & $6(31.6)$ & $7(30.4)$ & 1.000 \\
\hline $\begin{array}{l}\text { Median time to } \\
\text { recurrence } \\
\text { (range) }\end{array}$ & 7 months & 8 months $(5-37)$ & 8 months (5-37) & \\
\hline Progression (\%) & 0 & $1(5.3)$ & $1(4.3)$ & 1.000 \\
\hline Cystectomy (\%) & 0 & $4(21.1)$ & $4(17.4)$ & 0.562 \\
\hline Death (\%) & 0 & $1(5.3)$ & $1(4.3)$ & 1.000 \\
\hline
\end{tabular}

J Endolum Endourol Vol 2(4):e10-19; October 28, 2019.

This article is distributed under the terms of the Creative Commons Attribution-Non

Commercial 4.0 International License. (T) Tregunna et al. 


\section{Deaths}

Three patients (11.5\%) died during follow up, all from metastatic disease. Data were censored for the patient with no recent follow-up giving an overall Kaplan-Meier five-year survival rate of $88.6 \%$ (95\% CI 69.4-96.9\%). The patient who presented with node-positive UCB died eight months following diagnosis. The patient who progressed from non-muscle invasive UCB to nodepositive, locally advanced disease died 27 months after initial diagnosis.

The patient who presented with muscle invasive, node-negative UCB, micropapillary variant underwent upfront cystectomy and nephroureterectomy with G3 pT3b histology from the bladder present at the resection margin. This patient developed metastases two months later and died thirteen months after the original diagnosis.

\section{Comparison between Patients Aged Below 20 and between} 20 and 39 Years

Four patients (14.8\%) were diagnosed before the age of 20 years with a male to female ratio of 1:1. 23 patients $(85.2 \%)$ were diagnosed between the ages of 20 to 39 years with a male to female ratio of 3.6:1.

All patients in the youngest group had nonmuscle invasive UCB. Three of these $(75.0 \%)$ were stratified as low-risk, one (25.0\%) was intermediaterisk and none were high-risk. None had abnormal variant histology. Only one patient $(25.0 \%)$ had low-grade recurrences, none progressed, required radical treatment or died during follow up.

In the older group $(n=23)$ one patient $(4.3 \%)$ had muscle invasive, node-negative UCB, one patient (4.3\%) had muscle invasive, node-positive UCB and one (4.3\%) had epithelioid angiosarcoma. Both patients with variant $\mathrm{UCB}$ histology were in this group. A total of 20 patients had non-muscle invasive UCB $(87.0 \%)$. Of these patients, one did not have enough data to risk stratify therefore of the other nineteen patients, six $(31.6 \%)$ were risk stratified as low-risk and thirteen (68.4\%) high-risk with five being sub-stratified as highest-risk (26.3\%). Of the nineteen patients with complete follow-up data, six patients recurred $(31.6 \%)$ and one progressed (5.3\%). Overall, six patients with complete data (27.3\%) underwent cystectomy either upfront or due to high-grade non-muscle invasive UCB recurrence. A total of 3 patients $(13.6 \%)$ died from their disease.

\section{DISCUSSION}

Bladder tumours in patients $<40$ years of age are rare. We present, to our knowledge, the largest UK case series of such patients.

Age is the single greatest risk factor for the development of bladder cancer. ${ }^{9}$ It is thought that mechanisms for this are multi-factorial. In a review of the literature into the effect of age on bladder cancer Shariat et al. ${ }^{9}$ offered several theories for this.

These include:

- Cumulative exposure to environmental carcinogens as well as time for accumulation of cellular events which can lead to cancer development.

- Organ system deterioration reducing the ability to detoxify potential carcinogens.

- Increased incidence of bladder outflow obstruction with the reduction in the ability to fully empty the bladder, thereby prolonging contact between urinary carcinogens and urothelium.

- Decreased fluid intake with a resultant increase in the urinary concentration of such carcinogens.

- With ageing, cells may have a reduced ability to repair mutations in DNA that may lead to an increase in oncogene activity or a decrease in tumour suppressor gene activity.

- Outcomes for bladder cancer may differ in older patients as less aggressive therapies may be offered for treatment due to other co-morbidities. ${ }^{9}$

Bladder cancer is the fourth most common cancer in men accounting for $6.0 \%$ of all cancers. ${ }^{10}$ Generally, men are at a three to four-fold increased risk of developing bladder cancer when compared to women. ${ }^{9}$ In our series, the male to female ratio was slightly lower at 2.86:1 and although not statistically significant, an even lower male to female ratio was found in patients aged 20 years or less (M:F 1:1). In another retrospective comparison study of 50 patients with non-muscle invasive UCB aged under 45 compared with 90 patients aged over 55 years, the same finding was seen. In this study, the male to female ratio was 2.16:1 in the younger group and 7.18:1 in the older group. ${ }^{11}$ The authors hypothesized that this could be 
due to a more homogenous exposure to carcinogens in the younger group. ${ }^{11}$ Overall, however, males are more likely to develop bladder cancer even when adjusting for smoking and occupational exposure. ${ }^{12}$ By contrast, other studies of young patients with bladder cancer have found the male to female ratio to be as high as $10.4: 1^{13}$ with one meta-analysis demonstrating male dominance as more pronounced in a group diagnosed under the age of 20 compared with patients diagnosed between 30 and 40 years. ${ }^{5}$ It is unclear as to why such a difference is found and may be due to the retrospective nature of reporting in these studies.

In our group, $61.9 \%$ of patients presented with hematuria in keeping with $53.0-100.0 \%$ seen in other studies. $^{6,14,15}$ A total of two patients $(9.5 \%)$ presented as incidental findings on ultrasound scan. Certainly, in children and adolescents ultrasound is a useful diagnostic tool with $85.0 \%$ of tumours in children aged 9-16 years initially visible on ultrasound alone. ${ }^{15}$ In adults, ultrasound may not be as reliable due to structural changes such as trabeculations, although in one series of 31 patients aged under 40 years ultrasound was found to be diagnostic in all cases. ${ }^{16}$

$54.2 \%$ of our patients were cigarette smokers; smoking accounts for $50.0 \%$ of the attributable risk of bladder cancer. ${ }^{17}$ Our patients probably have a shorter history of smoking than older counterparts, and it is known that there is a 10 -year lag time from the development of lung carcinoma to developing UCB. This is thought to be since the bladder is exposed to more dilute carcinogens than the lung and therefore a longer exposure is required for mutations to occur. ${ }^{9}$ In our patients, however, smoking history probably remains relevant given the higher proportion of patients who smoked when compared to the general population, ${ }^{18}$ although we do not know the pack-year history for these individuals. Other studies of young patients with UCB have also found a high prevalence of smoking between $44.0 \%$ and $88.0 \%$. ${ }^{1,14,16,19,20}$

The histology from one patient in our study demonstrated epithelioid angiosarcoma. This patient was male and 31 years of age at diagnosis. He was treated initially with neo-adjuvant paclitaxel chemotherapy and went on to have a cystoprostatectomy with T0 histology. He was alive with no evidence of disease recurrence at 85 months of follow-up. Epithelioid angiosarcomas of the urinary tract are extremely rare and often associated with prior radiation therapy, although not in this case. They are aggressive, associated with a poor prognosis and progress rapidly with significant bleeding complications reported. ${ }^{21}$ From the few case reports in the literature the mean survival is 6 months (range $1.5-72$ months) ${ }^{22}$ indicating that this patient has survived much longer than expected for this disease.

In our series, $92.3 \%$ presented with the non-muscle invasive disease compared with $75.0 \%$ of patients with UCB in the overall population. However, these data are in keeping with multiple other studies of young patients who have demonstrated more than $90.0 \%$ of patients presenting with non-muscle invasive UCB. ${ }^{13,14,16,19,23}$ Some studies have, however, demonstrated more aggressive disease such as Ozbey et al., who in their series of 25 patients diagnosed $<$ 40 years of age over five years, found that $36.0 \%$ of patients were muscle invasive at diagnosis. ${ }^{20}$ The discrepancy in these data are difficult to understand as the majority of studies (including our own) are retrospective, lacking control populations and have relatively small numbers.

Contrary to published literature, a large proportion of our patients with non-muscle invasive UCB (56.0\%) presented with high-grade disease (G2 high-grade, G3 or CIS). Comparisons are difficult, however, due to the change in histopathological reporting over time with many papers only using the 1973 WHO classification of reporting grade 2 lesions and therein not distinguishing between low-grade and high-grade lesions. ${ }^{13,20,23}$ From published series which have used the 2004 WHO classification $13.4-32.0 \%$ of patients $<40$ years of age present with high-grade histology. ${ }^{1,6}$ Only one of our patients (4.0\%) presented with a papillary urothelial neoplasm of low malignant potential (PUNLMP), thus lower than in other published series of patients $<40$ years of age where $22.0-30.0 \%$ of patients present with this histology. ${ }^{1,6}$ This may be due to PUNLMPs not being correctly coded in our database as with other grades of UCB.

$61.5 \%$ of patients presented with pTa disease. This is similar to many other published reports of $58.0 \%-76.5 \%,{ }^{1,23,24}$ although a larger $(n=82)$ series of patients from China ${ }^{6}$ found a lower $\mathrm{pTa}$ rate of 
$24.4 \%$ with $61.0 \%$ of their patients having pT1 disease compared with our $23.1 \%$ which appears more in keeping to other reports. ${ }^{1,23,24}$

$7.7 \%$ of patients presented with muscle invasive disease with one $(3.8 \%)$ having node-positive disease. Other comparable studies have published muscle invasive figures of between $8.7 \%$ and $14.6 \% 0^{1,6,13,16,24}$ although one series of 40 patients demonstrated a rate of $2.5 \%,{ }^{19}$ with two other studies of 25 and 30 patients publishing muscle invasive rates of $36.0 \%$ and $20.0 \%$ respectively. ${ }^{20,25}$ Only one other series reported any patients with lymph node-positive disease: in a large French multi-center retrospective study of 142 patients < 40 years old with UCB, $14.0 \%$ had muscle-invasive disease, $7.0 \%$ had lymph node-positive disease and $5.0 \%$ had distant metastases. ${ }^{1}$

The published probability of recurrence at one year from non-muscle invasive UCB in patients of all ages is $15.0 \%-61.0 \%$ and of progression is $0.2-17.0 \%$, dependent on factors such as initial tumour size, multifocality, initial stage/grade, prior recurrence rate and the presence of CIS. ${ }^{8}$ Only two of our patients had less than 12 months of follow-up data. Overall, 30.4\% recurred with $71.4 \%$ of these doing so within the first year following diagnosis. $56.5 \%$ were originally stratified as high-risk of recurrence, and of these $38.5 \%$ recurred. Only one patient $(4.3 \%)$ who were originally sub-stratified as highest-risk progressed from their original stage of the disease. Other studies of patients $<40$ years have reported recurrence rates of $12.5-50.0 \%$ with progression rates of $0.0-19.0 \% .^{1,6,13,16,19,20,24,25}$ One New York study retrospectively compared 74 patients $<40$ years to a randomly selected cohort over 65 years of age treated over the same period. They showed that although older patients tended to have higher-grade tumours at diagnosis, there was no significant difference in disease-free progression or recurrence rate between the groups in patients with non-muscle invasive UCB with a five-year recurrencefree probability rate of $37.0 \%$ and $42.0 \%$ in young and older patients respectively. However, the authors noted that patients were all at an unexplained higher than usual risk of recurrence. ${ }^{26}$

Three of our patients (11.5\%) died during follow-up giving an overall Kaplan-Meier five-year survival rate of $88.6 \%$ (95\% CI $69.4-96.9 \%)$. Most other studies report all patients being alive at the time of publication. ${ }^{6,16,20}$ One 25-year cancer registry study in California reported a $58.0 \%$ reduced risk of bladder cancer death in younger patients. ${ }^{27}$ In the large French multi-centre study $6.0 \%$ of patients died during follow-up with a correlation between initial grade and stage. ${ }^{1}$ Another study of 30 patients reported a 5-year cancer-specific survival of $93.3 \% .{ }^{25}$ The Memorial Sloan Kettering cohort of 74 patients reported cancer-specific survival of $96.0 \%$ at seven years, compared with $90.0 \%$ in older patients. Despite significantly more older patients undergoing cystectomy ( $54.0 \%$ vs. $23.0 \%$ ) there was a significantly worse disease-specific survival in younger patients who required radical treatment due to occult metastatic disease, perhaps indicating a more aggressive subset of tumours in these patients. ${ }^{26} \mathrm{~A}$ poor prognosis was also seen in the patients undergoing immediate cystectomy for UCB in the French multi-centre study where three out of the seven patients $(42.9 \%)$ died within seven months of surgery. ${ }^{1}$ This finding was not found in another cohort of 82 patients from China where their cancer-specific survival was $100.0 \%$ during a median follow-up period of 90 months (range 12-186 months). However, in this study, despite 12 patients presenting with $\mathrm{T} 2$ disease only one of these patients was reportedly treated with any form of radical treatment (cystectomy). Whilst these data have been subjected to peer review the authors do not explain the difference between their outcomes and those of other groups. ${ }^{6}$

Our study compared patients under the age of 20 years with those aged between 20 to 39 . There were no statistical differences between the groups in terms of patient demographics, risk factors, tumour stage, rates of recurrence and progression or death. There was, however, a statistical difference in terms of higher-grade tumours being present in the older group $(p=0.026)$ as well as EAU risk-group stratification with more patients in the older group being stratified as high-risk of recurrence or progression compared with younger patients $(\mathrm{p}=0.009)$.

Elsewhere in the literature one study of 18 patients under the age of 30 years with UCB found a low $11.1 \%$ recurrence rate and a positive correlation between age and initial stage of the disease. ${ }^{28}$ A study of 8 patients diagnosed under the age of 18 years with 
UCB found no recurrence during a mean follow up of 15 years (range $8-27$ years). ${ }^{15}$ A study of 40 patients $(<40$ years) found that there was a significantly higher mean age of patients who developed recurrent UCB (34.8 vs. 28.5 years). ${ }^{19}$ Another study of 82 patients ( $<40$ years) found an overall recurrence rate of $23.2 \%$ but none in patients $<20$ years; with a significantly lower stage and grade at initial diagnosis compared with patients aged $20-40$ years. ${ }^{6}$ In the meta-analysis by Paner et al., ${ }^{5}$ patients were split into three groups, group one were less than 20 years old, group two aged 20-30 years, and group three aged 30-40 years. It was found that the incidence of grade 1 tumours decreased with increasing age decade, whereas higher-grade tumours increased. $94.0 \%$ of patients in group one presented with non-muscle invasive UCB compared with $80.4 \%$ of patients in group three. As well as this, rates of recurrence, progression, and death increased with age.

Many authors conclude that the difference in outcome may result from molecular differences between tumours in adolescents and very young adults when compared to tumours in older patients, although the 'cut-off' age is yet to be defined. Shelekhova et al., performed molecular subprofiling on 49 tumours and found significantly more aggressive molecular subtypes in patients between the ages of 30 and 45 compared with patients between 20 and 29 years. ${ }^{29}$ Owen et al., analyzed tumours in patients under the age of 19 years for alterations in DNA hypermethylation which can result in aberrant gene silencing, and compared these with matched lesions in patients aged 19 to 45 and also older patients with a median age of 78 years. They found significantly less methylation in the youngest group compared with the other groups, but no significant difference between the older two groups $(34.4 \%$ vs. $60.0 \%$ vs. $52.3 \%))^{7}$ Fibroblast growth factor receptor 3 (FGFR3) mutation and overexpression is seen in older adults with $\mathrm{UCB} .{ }^{4}$ Huang et al., looked at 45 patients aged 30 years or younger and found that positive immunoreactivity for FGFR3 was more common in patients aged 26-30 than in patients under 25 years ( $13.3 \%$ vs. $45.8 \%)$. In the older patients, there was a significantly higher recurrence rate suggesting a difference in molecular pathogenesis in younger patients. ${ }^{4}$ This was also observed in a study of 14 patients aged under 20 years were there was an absence of common mutations normally seen in UCB of older patients with no evidence of FGFR3 and chromosome 9 alterations. ${ }^{30}$

Whilst ours is the largest UK series of young patients with UCB the limitations of this study include the retrospective nature of analysis and small sample size, especially in our youngest cohort. However, our data support the importance of investigating young patients with symptoms such as hematuria. Future work would ideally include large, multicentre and prospective studies allowing the collection of a full dataset including the molecular analysis of tissue to identify genetic or epigenetic factors that may influence prognosis.

\section{CONCLUSIONS}

$\mathrm{UCB}$ is uncommon in patients under the age of 40 years but in symptomatic individuals remains an important differential diagnosis. The majority of patients have a good outcome, nevertheless, a proportion do present with aggressive disease and require radical treatment.

\section{REFERENCES}

1. Compérat E, Larré S, Roupret M, et al. Clinicopathological characteristics of urothelial bladder cancer in patients less than 40 years old. Virchows Arch 2015;466(5):589-94.

2. Beukers W, Hercegovac A, Zwarthoff EC. HRAS mutations in bladder cancer at an early age and the possible association with the Costello Syndrome. Eur J Hum Genet 2014;22(6):837-9.

3. Jayadpour N, Mostofi FK. Primary epithelial tumors of the bladder in the first two decades of life. J Urol 1969;101(5):706-10.

4. Huang H, Sun M, Li X, et al. Urothelial carcinoma of the bladder in patients aged 30 years or younger: clinicopathological analysis and expression of fibroblast growth factor receptor 3 (FGFR3) of 45 cases. Med Oncol 2015; 32(5):137.

5. Paner GP, Zehnder P, Amin AM, et al. Urothelial neoplasms of the urinary bladder occurring in young adult and pediatric patients: A comprehensive review of literature with implications for patient management. Advances in Anatomic Pathology 2011; 18(1):79-89. 
6. Wang ZH, Li YY, Hu ZQ, et al. Does urothelial cancer of bladder behave differently in young patients? Chin Med J (Engl) 2012;125(15):2643-8.

7. Owen HC, Giedl J, Wild PJ, et al. Low frequency of epigenetic events in urothelial tumors in young patients. J Urol 2010;184(2):459-63.

8. Babjuk M, Burger M, Compérat E, et al. Non-muscleinvasive Bladder Cancer (TaT1 and CIS) EAU Guidelines. 2019;1-50.

9. Shariat SF, Sfakianos JP, Droller MJ, et al. The effect of age and gender on bladder cancer: A critical review of the literature. BJU Int 2010;105(3):300-8.

10. Siegel RL, Miller KD, Jemal A. Cancer statistics, 2019. CA Cancer J Clin 2019;69(1):7-34.

11. Migaldi M, Rossi G, Maiorana A, et al. Superficial papillary urothelial carcinomas in young and elderly patients: A comparative study. BJU Int 2004; 94(3):311-6.

12. Hartge P, Harvey EB, Linehan WM, et al. Unexplained excess risk of bladder cancer in men. J Natl Cancer Inst 1990;82(20):1636-40.

13. Gunlusoy B, Ceylan Y, Degirmenci T, et al. Urothelial bladder cancer in young adults: Diagnosis, treatment and clinical behaviour. J Can Urol Assoc 2015;9(9-10):E727-E730.

14. Stanton ML, Xiao L, Czerniak BA, et al. Urothelial tumors of the urinary bladder in young patients: A clinicopathologic study of 59 cases. Arch Pathol Lab Med 2013;137(10):1337-41.

15. Bujons A, Caffaratti J, Garat JM, et al. Long-term follow-up of transitional cell carcinoma of the bladder in childhood. J Pediatr Urol 2014;10(1):167-70.

16. Nomikos M, Pappas A, Kopaka M-E, et al. Urothelial carcinoma of the urinary bladder in young adults: presentation, clinical behavior and outcome. Adv Urol 2011;480738.

17. Freedman ND, Silverman DT, Hollenbeck AR, et al. Association between smoking and risk of bladder cancer among men and women. JAMA - J Am Med Assoc 2011;306(7):737-45.

18. Cancer Research UK. Tobacco Statistics. Available at: https://www.cancerresearchuk.org/health-professional/ cancer-statistics/risk/tobacco.
19. Sen V, Bozkurt O, Demir O, et al. Clinical behavior of bladder urothelial carcinoma in young patients: a single center experience. Scientifica (Cairo) 2016;6792484.

20. Özbey I, Aksoy Y, Biçgi O, et al. Transitional cell carcinoma of the bladder in patients under 40 years of age. Int Urol Nephrol 1999;31(5):655-9.

21. Abbasov B, Munguia G, Mazal PR, et al. Epithelioid angiosarcoma of the bladder: report of a new case with immunohistochemical profile and review of the literature. Pathology 2011;43(3):290-3.

22. Nizam A, Paquette EL, Wang BG, et al. Epithelioid angiosarcoma of the bladder: a case report and review of the literature. Clin Genitourin Cancer 2018;16(6):e1091-95.

23. Mongiat-Artus P, Miquel C, Van Der Aa M, et al. Infrequent microsatellite instability in urothelial cell carcinoma of the bladder in young patients. Eur Urol 2006;49(4):685-90.

24. Erozenci A, Araus S, Pekyalcin A, et al. Transitional cell carcinoma of the bladder in patients under 40 years of age. Int Urol Nephrol 1994;26(2):179-82.

25. Wen YC, Kuo JY, Chen KK, et al. Urothelial carcinoma of the urinary bladder in young adults - Clinical experience at Taipei Veterans General Hospital. J Chinese Med Assoc 2005;68(6):272-75.

26. Yossepowitch O, Dalbagni G. Transitional cell carcinoma of the bladder in young adults: Presentation, natural history and outcome. J Urol 2002;168(1):61-6.

27. Lara J, Brunson A, Keegan THM, et al. Determinants of survival for adolescents and young adults with urothelial bladder cancer: results from the california cancer registry. J Urol 2016;196(5):1378-82.

28. Caione P, Patruno G, Pagliarulo V, et al. Nonmuscular invasive urothelial carcinoma of the bladder in pediatric and young adult patients: age-related outcomes. Urology 2017;99:215-20.

29. Shelekhova K V., Krykow KA, Mescherjakov IA, et al. Molecular pathologic subtyping of urothelial bladder carcinoma in young patients. Int J Surg Pathol 2019;27(5):483-91.

30. Wild PJ, Giedl J, Stoehr R, et al. Genomic aberrations are rare in urothelial neoplasms of patients 19 years or younger. J Pathol 2007;211(1):18-25. 